\title{
DIE BRASILIANISCHE MITTELSCHICHT UND DER ARCHETYP DES KLASSENVORURTEILS
}

\section{ORIGINAL-ARTIKEL}

GIESBRECHT, Daniel Florence ${ }^{1}$

GIESBRECHT, Daniel Florence. Die brasilianische Mittelschicht und der Archetyp des Klassenvorurteils. Revista Científica Multidisciplinar Núcleo do Conhecimento. Jahrgang 05, Ed. 02, Vol. 04, S. 104-124. Februar 2020. ISSN: 2448-0959, Zugangslink: https://www.nucleodoconhecimento.com.br/geschichte/archetyp-usvorurteile

\section{ZUSAMMENFASSUNG}

Dieser Artikel zielt darauf ab, eine historische Rekonstitution zu erarbeiten, aus der Langzeitserie, der Bildung der brasilianischen Mittelschicht und auch ihrem Archetyp us-Klassenvorurteile. Wir nutzten als Ausgangspunkt für unsere Reflexion die Tatsache, dass Brasilien mehr als dreihundert Jahre Sklaverei lebte, die die Fülle eines rassistischen Imaginären vermachte, was zu voreingenommenen Praktiken führte und zu afronachkommenden Bevölkerungsgruppen eingebürgert wurde, abgesehen davon, dass es sich auf den armen Bürger im Allgemeinen ausgedehnt hatte. Wir versuchen, die untersuchten Objekte auf historistische Weise mit den soziologischen Konzepten der Sozialisation in Beziehung zu setzen, neben der Charakterisierung der bürgerlichen Ideen der Mittelschicht und der brasilianischen Eliten aus der Geschichte der Mentalitäten. Wir beabsichtigen, zu einem besseren Verständnis der Hindernisse beizutragen, die durch das Fehlen von Andersseinpraktiken in den täglichen sozialen Beziehungen entstehen.

1 Doktorand in Zeitgeschichte; Master in Education; Studium der Sozialwissenschaften, Diplom in Pädagogik, Studium der Geschichte. 
Schlagworte: Soziale Klasse, Vorurteile, Sklaverei, soziale Ausgrenzung, Andersartigkeit.

\section{EINFÜHRUNG}

Als wir Wirtschaftsindikatoren über das brasilianische Potenzial untersuchten, stellten wir konsistente und hohe Zahlen fest, die im Vergleich zu anderen Ländern sehr wettbewerbsfähig waren, so sehr, dass Brasilien von den 1960er Jahren bis heute immer zu den fünfzehn Ländern mit dem höchsten Bruttoinlandsprodukt der Welt gehört.

Die Zahlen werden nicht wiederholt, wenn wir Referenzdaten über Armuts- und Ungleichheitsquoten suchen. Alarmierende Realitäten der Einkommensverteilung, hohe Gewaltraten, Kindersterblichkeit, unter anderem, werden leicht in den Augen derjenigen wahrgenommen, die durch das riesige brasilianische Territorium reisen und den Eindruck erwecken, durch zwei völlig unterschiedliche Welten zu schlendern: die der Opulenz und der des Elends, eine Illusion der Optik einer Wirtschaft, die erfolgreich in das Weltszenario eingefügt wurde, aber eine komplexe soziale Realität verschleiert. , die in ihrer Struktur Spuren einer tiefen Ungleichheit darstellt.

Wir werden das Wort "Armut" verwenden, um auf die Situation von Millionen von Menschen in Brasilien zu verweisen. Aber das Konzept der Armut wird, abhängig vom verwendeten Kontext und seinem semantischen Factoring, komplex und polysemic. Aus dieser semantischen Komplexität wollen wir unsere Reflexion durch diesen Artikel ableiten, der die Armut auf den Archetypus der Bildung der brasilianischen Mittelschicht und ihre Klassenbeziehungen bezieht.

Im intrinsischen Imaginären der brasilianischen Mittelschicht in ihren Kreisen der Sozialisation wird die Bildsprache der Armut in eine Wahrnehmung verklärt, die so vereinfacht ist von der Wirklichkeit, die sie umgibt, bis zu dem Punkt, dass das Individuum der subalternen Klasse, oft in einem Zustand der Unsicherheit, unbewusst den Diskurs der Klasse erwirbt, zu der es nicht gehört und durch die es ausgebeutet wird. In dieser Situation ist es nicht schwer, auf alltägliche Phrasen des reinsten 
gesunden Menschenverstandes zu stoßen, die jederzeit eine Erklärung für die Missstände des Landes suchen: "Er ist arm, weil er ein Vagabund ist", "Arm arbeitet nicht gerne" oder, noch, "Arme wissen nicht, wie man etwas tut und kann nichts lernen".

Phrasen wie diese führen am Ende zu einer Einbürgerung des Sozialen, das heißt, sie klammert sich an den historischen und sozialen Charakter einer Tatsache, die sie auf ein natürliches Ereignis reduziert. So hört so etwas wie Mangel auf, als Ergebnis einer von vornherein zusammengesetzten Struktur gesehen zu werden, die ungleichheiten in ihrer Praxis legitimiert, um als persönliches Attribut eines Individuums betrachtet zu werden.

Unter Bezugnahme auf die Idee der Einbürgerung von Ungleichheiten und Individualisierung von Erwartungen und Misserfolgen ad hoc, ist es notwendig, eine genaue Reflexion der Klassenfaktoren, die zur Einbürgerung des Archetyps der Minderwertigkeit von sozialen Gruppen im Nachteil beitragen und die vorgefassten Modelle seit dem Aufkommen des historischen Positivismus des neunzehnten Jahrhunderts bricht, dass, umwandlung der Sozialwissenschaften in die Natur durch unveränderliche Gesetze, die menschliche Kontrolle entkommen , induzieren Konformismus, passive und resignierte Akzeptanz dessen, was historisch und sozial produziert ist (Sucupira, 1984). Der Archetyp der Armen, die täglich scheitern, so verwurzelt im brasilianischen Kollektiv, das von der Kolonie bis zur Zeitgenossenschaft imaginiert ist, ist in diesem Sinne eine ideologische Operation, die dazu dient, eine bestimmte Gesellschaftsordnung aufrechtzuerhalten, d. h. eine Konfiguration des wirtschaftlichen Lebens, die den sozialen Klassen zugute kommt, die diese Ideologie hervorbringen.

Wir werden zunächst Souzas theoretischen Rahmen (2019) verwenden, der der Kritik an den Paradigmen, die das brasilianische Sozialdenken leiten, insbesondere dem des "Kulturalismus", in seinen Versuchen, die Ursprünge der Ungleichheiten im Land zu erklären, mehr anzutreffen schien. Es ist auch unsere Absicht, dem Leser die Ideen vorzustellen, dass wir a posteriori verteidigen werden, insbesondere, dass die Strukturierung des mentalen Archetyps der brasilianischen Mittelschicht eines der 
Haupthindernisse für den Aufbau eines Landes ist, das die republikanischen Werte festigt, und das die Bedingungen für die Ausweitung der Bürgerrechte für Millionen seiner Einwohner schafft.

\section{POLISSEMIA DER ARMUT}

Armut ist ein historisches und soziales Phänomen. Das bedeutet, dass es eng mit der Struktur der Gesellschaft und der Art und Weise verbunden ist, wie jede soziale Klasse die produzierten Waren oder den erzeugten Reichtum anpasst. Es ist kein einfaches Konzept, aber wir können verschiedene Kriterien verwenden, um zu versuchen, es zu definieren.

Das Kriterium des persönlichen oder familiären Einkommens wurde in Brasilien am häufigsten verwendet, aber wir glauben, dass es aufgrund vieler Faktoren, die sich in den letzten Jahren noch verschlimmert haben, ungenau ist, wie das Wachstum informeller Aktivitäten, die eine effektive Kontrolle des Einkommens verhinder[2]n.

Neben dem Einkommen besteht ein weiteres Kriterium zur Konfiguration der Armut darin, zu überprüfen, was für die Reproduktion des Lebens als wesentlich erachtet wird. Dieses Kriterium ist daher unserer Ansicht nach auch deshalb reduktionär, weil es aufgrund internationaler Übereinkommen Armut nur mit dem Lebensmittelkonsum in Verbindung setzt.

Es erscheint sinnvoller, das Konzept auf "Grundbedürfnisse" auszudehnen, die nicht nur auf Lebensmittel beschränkt wären, sondern andere Aspekte umfassen würden, die für die materielle Reproduktion der Existenz als unverzichtbar erachtet werden, und die Idee der Armut für die Unfähigkeit von jemandem oder einer Familie, Mittel zu haben, die den Zugang zu einer Reihe von Gegenständen ermöglichen, die für das Überleben als unerlässlich erachtet werden, erweitern. Dies würde auch konfiguriert werden, wenn die Person oder Familie aus dem Bildungssystem ausgeschlossen ist, da die Schule heute unerlässlich ist, um in den Arbeitsmarkt einzutreten. Nach Studien von Rocha (2006) konnten wir erst mit der Erweiterung dieser Konzepte ein Land von 
kontinentaler Dimension wie Brasilien verstehen, das in enorme regionale Disparitäten versunken ist.

Unsere argumentative Idee ist es, die materielle Auffassung der Konstitution des Armutsbegriffs für eine abstrakte und unbewusste psychologische und strukturelle Verwertung genau zu überwinden, die in der brasilianischen historischen Konstruktion dieses Konzept legitimiert, das in einem methodischen Rahmen verankert ist, der durch den Archetyp des Vorurteils gestützt wird, der soziale Gruppen in einem Land minderwertig, das seit mehr als dreihundert Jahren die Tagesmarken einer Wirtschaft und Gesellschaft hat, die auf Kosten der Sklavenarbeit orientiert sind.

Vorurteile können verschiedenen materiellen Interessen dienen, die sein können: Klasse, Geschlecht, politische, rassische oder ethnische und religiöse. Aber um die Konstitution von Vorurteilen zu demonstrieren, die von der brasilianischen Mittelschicht eingebürgert wurden, müssen wir zweifellos die historischen Strukturen kennen, die durch die vorkapitalistische Vergangenheit die Abstände zwischen den sozialen Gruppen einer Gesellschaft synthetisierten, die sich von der Bipolarität zwischen Ihnen und dem Sklaven in Richtung einer komplexeren Organisation mit dem Aufkommen des Handels- und Industriekapitalismus bewegte, aber immer den Archetyp usamerikanischer Vergangenheit bewahrt hat.

Diskriminierung und Vorurteile können auch Gefühle der Resignation und Konformität bei den Opfern hervorrufen, die akzeptieren, unterdrückt und ausgebeutet zu werden, wie Sartre (1905-1980) in einem Theaterstück mit dem Titel The Respectful Prostitute (1946[3]) warnte. Es scheint uns der brasilianische Fall zu sein, mit den täglichen Einstellungen seiner Mittelschicht der Minderwertigkeit der weniger Begünstigten, was sie dazu veranlasst, ihre eigenen Vorurteile und die Idee des Scheiterns zu introjekten, weil sie glaubten, dass sie solche Diskriminierungen verdienen. 


\section{HISTORISCHE URSPRÜNGE DER VORURTEILE DER BRASILIANISCHEN MITTELSCHICHT}

Zwischen dem 16. und 19. Jahrhundert verband der afrikanische Sklavenhandel mit Amerika die beiden Kontinente mit dem lukrativsten Handel in der brasilianischen Geschichte. Afrikanische Sklaven waren bei den unterschiedlichsten Alltagsaktivitäten präsent. Die Einbürgerung der Sklaverei ist zu etwas geworden, das so in den Strukturen der Gesellschaft verwurzelt ist, dass es sehr unwahrscheinlich ist, dass sie ohne die traditionellen Spaltungen derer, die befehligten und gehorchten, vorstellbar wäre. Dieses autoritäre Modell der sozialen Beziehungen ermöglicht das Verständnis der symbolischen Beständigkeit, die gegen die ärmsten Bevölkerungsgruppen praktiziert wird, auch nach der Abschaffung der Sklaverei am Ende des 19. Jahrhunderts.

Mit der Entwicklung des brasilianischen Sozialdenkens, vor allem seit Beginn des 20. Jahrhunderts, haben wir versucht, das dichotome Bild zu erklären, das die einheimische Gesellschaft im Laufe ihrer Geschichte erlebt hat. Wir glauben, dass für diese Aufgabe die am besten geeignete Theorie, die Förderung eines besseren Verständnisses der Konstruktion dieser sozialen Struktur, ist, dass der seriellen Geschichte, in fernand braudel Smethodologien vorhande[4]n. Eine semiotische Analyse der Bildung brasilianischer Sozialklassen ist nur möglich, wenn wir die Serie der langen Dauer der Sklaverei wahrnehmen, die in der täglichen Mentalität des Landes den Archetypus der Vorurteile, der Trennung und der Segregation, von casagrande zu senzala bestätigte, die mit dem Übergang zur Moderne der kaufmännischen und industriellen Phase des Kapitalismus eine neue Form der Ausbeutung bestätigten. , aber mit der strukturellen Kleidung der alten, segregierenden Reichen und Armen in den großen Städten, sowie in sozialen Kreisen.

Es sei auch daran erinnert, dass die Entwicklung der Klassen in Brasilien, nach Fausto (1997), mit der Konsolidierung einer proprietären Klasse zusammenhängt, die Landbesitz und andere Produktionsmittel besitzt. In einem Land, das einst 
grundsätzlich landwirtschaftlich war, eignete sich diese Klasse leicht den größten Teil des produzierten Reichtums, da sie die versklavte Arbeit ausnutzte.

Unter der Schirmherrschaft der Kolonialgesellschaft entwickelte sie sich in ganz besonderer Weise in den Regionen, in denen das Plantagensystem konsolidiert wurde, soziale Beziehungen außerhalb der Sklaverei zwischen Freigelassenen und Eigentümern. Mit dem Fehlen eines direkten kolonialen Systems, zusätzlich zu einer territorialen Unermesslichkeit, die die Umsetzung optimierter Formen der Kontrolle unter der Vormundschaft der portugiesischen Krone behinderte, und der Schaffung des Systems der Verteilung erblicher Kapitäne - ein Versuch einer öffentlich-privaten Schlichtung im Kolonisationsprozess - wurden mehrdeutige, widersprüchliche und deregulierte Formen der Beziehungen zwischen Individuen verschiedener Klassen entwickelt. In diesem Szenario finden wir die Zahl des Aggregats.

Diese Zwischenschicht formal freier Menschen, zwischen Meistern und Sklaven, wird von abhängigen Männern und Frauen gebildet, sowohl materiell als auch symbolisch von Grundbesitzern und Menschen. Im ökonomischsten und materiellen Sinne ist die Abhängigkeit dieses sozialen Extrakts unaufhaltsam. (...) Dies ist unter uns - als wirtschaftliche, politische und soziale Form - die Figur des "Aggregats". Als grundlegende Figur in der brasilianischen Literatur und historischen Soziologie wird das Aggregat die erste Zwischenklasse zwischen Eigentümern und Enteigneten bilden. (Sousa, 2018: 65-66).

Obwohl sie noch weit von der Entstehung der modernen brasilianischen Mittelschicht entfernt ist, ist es von größter Bedeutung, die geistige Introziation von Vorurteilen zwischen den zukünftigen sozialen Klassen des modernen Brasilien zu verstehen, da diese innerhalb eines Systems autoritärer Herrschaft der privaten Macht über die öffentliche, war es genau die Kaste, die die Prinzipien der Kontrolle und Gewalt einbürgert, die in gewisser Weise in gewisser Weise sadistisch von den sklavenarbeitenden ländlichen Eliten praktiziert wurden, die auch den Aggregaten die Rechte delegierten, diejenigen zu unterdrücken, die als "Instrumente, die sprachen", bestialisiert und entmenschlicht angesehen wurden. 
Die Tatsache, dass uns von hier aus interessiert ist, ist zu erkennen, dass es durch dieses Verhältnis der Interdependenz zwischen Denherren und Aggregaten, zusätzlich zu dem Unterstaatlichen als Organisator der Gesellschaft errichteten Gewaltmonopol war, dass das Gefühl der Verachtung für den anderen zur Konstitution sadistischen Verhaltens mit den Versklavten und Demütigsten führen sollte. Die Freude an Gewalt wird in der Geschichte dieser Zwischenklassen in der brasilianischen Gesellschaft allgemein bestätigt, bis hin zu intensiveren - dort empfinden wir Sadism[5]us als eine Form der Gruppenanerkennung oder Überlegenheit über die andere - als eine allägliche Praxis derer, die wir freie Männer nennen. Szenen von Demütigung, Witzen, Satiren und stumpfem Verhalten werden Teil des Behandlungsumfangs der Marginalisierten sein, die wir als sozialen "Scum" bezeichnen werden.

Seit Beginn des Bergbaus in der Dämmerung des siebzehnten Jahrhunderts und während des achtzehnten Jahrhunderts erlebte Brasilien das Phänomen der Urbanisierung, das sich hauptsächlich auf die Kapitänsrolle von Minas Gerais konzentrierte. Die geschichtete Gesellschaft des Nordostzuckers war die gesellschaft mit einem dynamischeren und facettenreicheren Charakter in Vila Rica. Sklaverei und Freiheit gingen in einer interdependenten Wirtschaft Hand in Hand, aber sie bot neue Formen des sozialen Aufstiegs, einschließlich schwarzer Liner ${ }^{[6]}$. Die Bergbauerfahrung war eigenartig, in dem Mestizen, Schwarze und Indianer einen einzigartigen Moment hatten, in dem sie zum ersten Mal ihre Talente, daher die große Anzahl von Künstlern, Handwerkern, Buchhaltern und das Auftreten mehrerer anderer Berufe in der Region in die Praxis umsetzen konnten. Zum ersten Mal in der Geschichte Brasiliens schien sich ein ausdrucksstärkeres technisches Wissen von einer Gesellschaft zu unterscheiden, die durch "rohe" und manuelle Arbeit aufgebaut wurde und den Weg für die Entstehung der modernen Mittelschicht ebnete.

Das frühe 19. Jahrhundert war der Vorbote neuer Luft, die den europäischen Kontinent erschüttern würde - und sein größter Sturm war Napoleon Bonaparte. Die vom französischen Kaiser initiierten Kriege wurden neben seinen größenwahnsinnigen Ambitionen mit der neuen Form in Verbindung gebracht, die das kapitalistische System in seiner industriellen Phase angenommen hatte. Frankreich, Großbritanniens 
historischer Feind, begann einen Konflikt kontinentalen Ausmaßes, um seinen gerechten Anteil zu erobern, in einem Omen für das, was das imperialistische Phänomen sein würde, das noch in der Mitte des Jahrhunderts ausgelöst werden sollte. Diese Tatsache hätte direkte und indirekte Auswirkungen auf die Zukunft Portugals und damit Brasiliens, da die städtische Verwaltungsstruktur die Meere überqueren würde, um sich in der Kolonie zu etablieren.

Dieser Prozess, den die portugiesische Königsfamilie zu konsolidieren kam, war bereits bei der Entdeckung von Minen in Gegenwart einiger kolonialer Ausdrucksstädte vorgesehen, in der Notwendigkeit einer größeren Wachsamkeit gegenüber neu entdecktem Reichtum und einer größeren Kontrolle, von da an, auf Familismo und privaten Mandonismus. Ein symptomatisches Beispiel für den Übergang der Macht vom Land in die Städte ist der Fall der Schulden der bisher uneinbringlichen ländlichen Patriarchen, die fortan unter Polizeidienst bezahlt werden. So wichtig der Wandel vom wirtschaftlich dynamischen Zentrum des Nordostens zum Südosten war auch die gesellschaftliche Transformation großer Proportionen, die neue Gewohnheiten, neue soziale Rollen, neue Berufe und letztlich den Aufbau einer neuen sozialen Hierarchie implizierte. (Souza, 2019: 61).

Die Urbanisierung stellte schließlich eine Größenänderung der Machtstrukturen dar, wodurch sie vom individuellen Patriarchalismus in die Hände des organisierten Staates überging. Aus Weber-Sicht begann sich das legitime Gewaltmonopol auf die Hände des Staatsapparates zu konzentrieren - wie zum Beispiel die Polizei -, die sich mit den Vorrechten der alten Formen des Klassenautoritarismus verschmolzen, aber die Praxis des Sadismus aufrechtzuerhalten, die früher privat von öffentlichen Kräften verübt wurde.

Mit der Öffnung der Häfen für befreundete Nationen im Jahre 1808, die vom damaligen Prinzregenten D. Joo VI. angeordnet wurde, und mit der Unterzeichnung der Handelsund Schifffahrtsverträge von 1810 würde Brasilien Veränderungen erleben, die sich sofort auf seine koloniale Struktur auswirken würden. Die Umgebung der Hafenstädte, 
vor allem Rio de Janeiro, Salvador und Recife wurde, über Nacht, geschäftige Zentren des Handels, mit dem Eintrag von unbekannten Produkten bis dahin, die die Bildung einer monetären Struktur gefördert. Wechsel- und Handelsbüros, Import- und Exportagenturen, Banken, kurz gesagt, eine Vielzahl von Aktivitäten, die bisher in der Welt des ländlichen Patriarchats bisher unnötig waren, nur in ihrem richtigen Ausmaß in Bergbauregionen erlebt[7].

Aber der große soziale Wandel, der durch dieses neue europäisierte Szenario der Wirtschaftsbeziehungen herbeigeführt wird, wäre die Einreise von Tausenden von Einwanderern. Neben den rund 15.000 Portugiesen, die gemeinsam die königliche Familie ankamen, kamen auch Engländer, Deutsche und Franzosen aus wirtschaftlichen bis künstlerischen Gründen nach Brasilien. Das zukünftige Land, das 1822 geboren werden sollte, markierte bereits Merkmale eines unabhängigen Staates.

Immigranten waren freie Arbeitskräfte, die, zusätzlich zu den in Brasilien geborenen freien Arbeitern, die moderne Mittelschicht bilden würden, über die wir vorhin gesprochen haben. Eine andere Klasse als der alte ländliche Haushalt, der den mittleren Teil der sozialen Hierarchie besetzt, aber ohne die Notwendigkeit für den Schutz seines Kompads. Liberale Prinzipien und Individualismus, die seit dem 18. Jahrhundert mit der Philosophie der Aufklärung aufgeblasen worden waren, traten ins Unbewusste des Stadtbewohners ein. Eine Art selbstgemachter Mann der Tropen würde das Imaginäre der Stadt bevölkern.

Mit dem Kaufalter öffnet der Kapitalismus ein grundlegendes Vakuum für seine Erhaltung und Reproduktion: die Notwendigkeit des kulturellen Kapitals, es zu betreiben. Kommerzielle, verarbeitende, bürokratische und lehrmedizinische Tätigkeiten - um nur einige Beispiele zu nennen - erfordern eine Form von technischem und utilitaristischem Wissen für ihren Betrieb, eine Tatsache, die zum Widerstand der manuellen Arbeit beitragen würde, die bisher häufig vom Sklaven oder den Ärmsten durchgeführt wurde. Die Mittelschicht wird die Gruppe sein, die sich dieses Wissen aneignen wird und sich durch sie von den Bescheidensten, denen schwerer Arbeit, von der Ebene der Tiere distanzieren wird. 
Für die Verlassenen und Vergessenen bleiben die disqualifizierten Aufgaben, die typisch für das Geplänkel sind. Diese werden, wie im Falle der Hausarbeit, weitgehend eine Fortsetzung der Sklaverei sein, die jetzt unter neuen Masken steht. Die direkte Nutzung von Muskelenergie - die uns kaum von einem Pferd oder Maultier unterscheidet - mit wenig eingebautem Wissen ist das unverwechselbare, wenn nicht das einzige Merkmal in Bezug auf Arbeitnehmer mit einem höheren Grad an technischer Qualifikation. (Souza, 2018: 74).

Sowohl diese neue Mittelschicht als auch die zukünftige minimal spezialisierte Arbeiterklasse, die wir "niedrige Mittelschicht" oder "proletarisch" nennen werden, werden um jeden Preis versuchen, sich von der Bevölkerung abzugrenzen, die hauptsächlich von Schwarzen und Halbrassen gebildet wird. Da sie nicht über die Produktionsmittel verfügen, werden sie zumindest versuchen, die symbolischen Werte der Eliten nachzuahmern, indem sie über die Besessenheit von der Europäisierung der Bräuche und durch formale und technische Bildung in der Vongeordnetheit von der Handarbeit nachdenken, die von westlichen Werten so abgeschrieben wird. Hier haben wir unsere erste konkrete Manifestation von Vorurteilen, eingebettet durch die moderne Mittelschicht des 19. Jahrhunderts.

Endogene und exogene Fragen im Zusammenhang mit der brasilianischen Sklavenfrage waren entscheidend für die anfängliche Reifung des Prozesses der Modernisierung der Arbeitsbeziehungen und die Reifung des Industriekapitalismus im 19. Jahrhundert. Seit der Unabhängigkeit Brasiliens drängt England den neugeborenen Nationalstaat, Verpflichtungen zum schrittweisen Aussterben des seit Mitte des 16. Jahrhunderts praktizierten Sklavenhandels unter Beweis zu stellen. Interne Bewegungen, die von liberalen Intellektuellen angeführt werden, wie die Kaffeeelite selbst, debattierten offen über die Abschaffung.

Solche Tatsachen könnten zunächst auf sui generis Weise darauf hinweisen, dass das Land in seinem Tempo seinen Übergang vom kommerziellen zum industriellen Kapitalismus konsolidieren könnte, weil das Szenario, das mit der Abschaffung der Sklaverei eröffnet wurde, nicht nur eine Ungeheuerlichkeit des Kapitals in die 
produktiven Sektoren verdrängen würde, sondern auch die Modernisierung der Klassenverhältnisse, die die Amalgame des veralteten Sklavensystems überwinden würde. Aber das ist nicht passiert. Die Industrialisierung würde sich erst im folgenden Jahrhundert unter der Leitung des Staates, in den Regierungen Getélio Vargas und Juscelino Kubitschek sowie in der diktatorischen Periode konsolidieren. Die Überwindung der Sklaverei auf inrem symbolischen Gebiet würde niemals überwunden werden.

Selbst als die Abschaffung 1888 vollzogen wurde, eine Tatsache, die Brasilien zum letzten Land der Welt machte, das die formelle Sklaverei beendete, setzte sich das perverse Szenario physischer und symbolischer Gewalt fort. Darüber hinaus stießen befreite Schwarze auf enorme Schwierigkeiten bei ihrer Eingliederung in den Arbeitsmarkt, da sie immer noch mit den zugewanderten Arbeitskräften konkurrieren mussten, die nun bewusst als staatliche Praxis angeregt werden. Es war in diesem perversen Verhältnis der Beständigkeit, dass im Laufe der Zeit, Brasilien gebildet wurde, im Zusammenhang mit dem Urbanisierungsprozess, eine Klasse der Bevölkerung, die völlig von den Grundprinzipien der Staatsbürgerschaft ausgeschlossen wurde, und[8] dies würde auch seine nächsten Generationen beeinflussen, ein Effekt, den wir den "trüben Schneeball" nennen werd[9]en.

\section{OHNE INDUSTRIALISIERUNG UND DAS BÜRGERLICHE IMAGINÄRE DER MITTELSCHICHT}

Die alte Rassenordnung verschwand nicht mit dem Ende der Sklavenarbeit. Stattdessen wurde sie assimiliert und in die neuen sozialen Strukturen integriert, die sich aus der Entwicklung der freien Arbeit ergeben. Fernandes (1974) zufolge profitierte die mit dem Ende der Sklaverei ausgelöste bürgerliche Revolution nur von einigen Teilen der brasilianischen Gesellschaft.

$\mathrm{Zu}$ den am meisten bevorzugten gehörte der "weiße" Teil der Bevölkerung europäischer Herkunft, der die Theorien des Bleichens im Land bestätigte. Diese Gruppe profitierte von allen Transformationen, die sich aus dem Wirtschaftswachstum, dem Industrialisierungsschub, dem Urbanisierungsprozess und der Entwicklung einer 
Stadtkultur ergaben. Die marginalisierte Klasse, die sich aus Afronachkommen zusammensetzte, blieb am Rande der nationalen Gesellschaft, weitgehend von allen Verbesserungen ausgeschlossen, die diese Transformationen hervorbrachten. Diese Tatsachen wiesen darauf hin, dass die Entsendezeit der Sklavenarbeit in den letzten Jahrzehnten des 19. Jahrhunderts nicht mit wesentlichen Veränderungen in der alten Rassenordnung einherging, die von Vorurteilen und Diskriminierung geprägt war. Die soziale Marginalisierung von etwa $50 \%$ der Brasilianer hat deutlich die Grenzen der wirtschaftlichen Modernisierung des Landes deutlich gemacht.

Mit der Aufrechterhaltung der Produktionsmittel in den Händen der Agroexportelite und der beginnenden Industrieklasse wurde in der Stadt und in ihrem kosmopolitischen Umfeld der bürgerliche Lebensstil und mit inm seine ganze Symbolik gefestigt. Mit dem Aufkommen der städtischen Mittelschicht, vor allem der auf intellektuelle und finanzielle Aktivitäten ausgerichteten, verbreitete sich das Gefühl der Europäisierung und der Bräuche. Die Einbeziehung europäischer aristokratischer Werte kann in der Annahme eines Musters von Geschmack und Luxus wahrgenommen werden, das dem der aristokratischen Schichten entsprechen sollte. In einer Art "symbolischem Kannibalismus" hat die wohlhabendere Mittelschicht Vorurteile und Patriarchate introjeciert, aber mit einer Kleidung, die als modern galt.

In einer symbolischen Dialektik ist die Mittelschicht eines der besten Elemente für uns, um wahrzunehmen, wie die öffentliche und private Sphäre grundlegend für ihre Selbstbestätigung, Anerkennung und Unterscheidung war. Auch weit entfernt vom modernen Konzept der "Meritokratie" [10]nimmt man wahr, wie der Archetyp der "gut angepassten" im Imaginären des Durchschnittsbürgers widerhallt. Merit ersetzte das Geburtsrecht als eine neue Form der sozialen Differenzierung sowie intellektuelle Raffinesse, ein weiteres Element, das begann, die Mittelschicht von den Volksschichten zu unterscheiden, noch ohne Zugang zu freier und hochwertiger öffentlicher Bildung.

Die Verallgemeinerung der Bürgerlichen Familie, in der sich die Frau um ihre Kinder und Hausarbeiten kümmerte - die die Arbeit zahlreicher Mitarbeiter kontrollierte - und der Mann, der an den Aktivitäten der Geschäftswelt teilnahm, ist in diesem Sinne einer 
der auffälligen Aspekte, denn sie gibt dem Einzelnen das Gefühl der Kontinuität, indem er bei seinen Kindern die Möglichkeit sieht, mit dem Familienbetrieb fortzufahren. Daher war die Einbeziehung aristokratischer Werte, mehr als bloße Modeerscheinung, Teil des Prozesses der Konsolidierung der politischen und sozialen Hegemonie und der Weltanschauung der Mittelschicht über die Gesellschaf[11]t.

Sobald das bürgerliche symbolträchtige Universum der brasilianischen Mittelschicht etabliert ist, wird die antagonistische Wahrnehmung dieses gegenüber den anderen weniger wohlhabenden Gruppen und inr Versuch, sich symbolisch mit den Eliten auszustatten, diese ziemlich wirklichen Besitzer der Produktionsmittel und der wirklichen Kontrolle des Landes, deutlich. Diese Besessenheit, Eliten zu ähneln, wird, außer in einigen Fällen, nicht in der Cash-Sphäre anerkannt werden, daher bleibt ihre symbolische Gleichwertigkeit durch sozialen Status oder[12] Prestige bestehen, und dies kann durch drei Elemente erlangt oder anerkannt werden: produktive Beschäftigung (Beschäftigung), Konsum und Lebensstil, die alle voll und ganz für die moderne kapitalistische Gesellschaft geeignet sind, oder, mehr als das, , eine Ergänzung zur anderen.

Mit der Eroberung des Status in der modernen brasilianischen MeritokratieGesellschaft werden neue Formen des Prestiges Teil der neuen sozialen Hierarchie. Das Wichtigste für die Mittelschicht ist neben der "Tugend" der Nachahmung, sich gegenseitig von den täglichen Praktiken des sozialen "Scum" zu distanzieren. Wein trinken auf Kosten der Kachaa, klassische Musik statt populärer Musik hören, Theater, Literaturakademien und teure Restaurants für den Exhibitionismus der Etikette besuchen, zusätzlich zu internationalen Reisen - mit dem Recht auf Beratung durch Pseudointellektuelle, die das zweideutige Gefühl der Abscheu vor dem Nationalen nähren - sind kleine Beispiele für diese reduktionistische Weltanschauung.

\section{KULTURKAPITAL, SOZIALE INSTITUTIONEN UND VORURTEILE}

Anders als die kulturistische Sicht, die am Anfang unseres Textes als eine Möglichkeit identifiziert wurde, soziale Ungleichheiten und Klassenverhältnisse zu erklären, Konzepte zu subjektivieren und Unterschiede nur aus individueller materieller Sicht zu 
rechtfertigen, müssen wir vom soziologischen Verständnis der primären und sekundären Institutionen der Sozialisation ausangehen, um das Phänomen des Klassenhasses zu verstehen, das im Alltag so präsent ist.

Im Allgemeinen beginnt die Sozialisation in der Familie, einer Institution, in der der Einzelne die Sprache lernt und durch sie kommuniziert, Bedürfnisse sowie Gefühle des Unbehagens oder des Wohlbefindens zum Ausdruck bringt. Psychologische Forschungen wie piaget (2005) bestätigen die Bedeutung von Eltern und anderen Familienmitgliedern bei der Bildung der Persönlichkeit der Kinder. Diese Studien zeigen, dass Familienmitglieder zu Beginn des Sozialisationsprozesses tendenziell entscheidender sind als die Personen, mit denen das Kind nur gelegentlich in Kontakt kommt. Wir finden hier ein Problem: In der Organisation der brasilianischen Sklavengesellschaft wurde alles getan, um den Aufbau von Solidaritätsbanden zwischen den versklavten Bevölkerungen zu vermeiden, und die erste Institution, die gekämpft wurde, war die Familie, die diese Phase der Sozialisierung kompromittiert. Bemerkenswert ist, dass es kaum Raum für die Konstituierung von primären sozialen Bindungen im Gefängnis gab, ohne die psychischen und emotionalen Bedürfnisse von Gefangenen zu berücksichtigen.

Die Schwierigkeit der Familienorganisation ist kein bloßes Phänomen der Sklavenvergangenheit, sondern bleibt zu gegebener Mehrheit bei den Kindern, Enkeln und Urenkeln der früheren Erniedrigungsgenerationen unter den bescheidensten Bevölkerungsgruppen. All dies wird tiefgreifende Folgen in zukünftigen sekundären Beziehungen haben, insbesondere im Schulunterricht, was zu geringeren Prestigechancen auf dem Arbeitsmarkt führen wird. Schwächere Familien werden in der Regel von den Oberschichten als "misfit" besteuert, insbesondere durch den generalistischen Diskurs der Mittelschicht, die von der Wiege aus Solidaritätsbande aufbauen konnte, die für den zukünftigen Erfolg entscheidend sein werden.

Der aktuelle Fall der Ausbeutung des brasilianischen Tollpatschs, um Zeit vor schmutzigen und schweren Hausarbeiten zu sparen - die es Ihnen ermöglicht, die "gestohlene" Zeit zu einem abscheulichen Preis für produktivere und gut bezahlte Aktivitäten zu nutzen - zeigt eine klare 
Elendsfunktionalität wie Sonnenlicht. Dieser stille Klassenkampf befreit eine ganze Klasse von Kinderbetreuung und Heimleben, wodurch Zeitsparendes Geld und qualifiziertes Lernen verändert wird. Die gestohlene Klasse ist in diesem Fall auf ewig dazu verdammt, die gleichen weltlichen Dienstaufgaben zu spielen. (Souza, 2019: 85).

Sparen Sie Zeit bei manuellen Aufgaben und investieren Sie Zeit in intellektuelle Aktivitäten. Infolgedessen eine größere berufsausbildungliche Ausbildung und damit ein größeres soziales Prestige; Es wird ein Kreis gebildet, der nichts mit Verdienst oder persönlicher Leistungsfähigkeit zu tun hat, sondern mit der Aneignung eines größeren kulturellen Kapitals.

Laut Bourdieu (2004) haben Kinder aus wohlhabenden Familien tendenziell höhere Schulleistungen als Jugendliche aus Familien, die zu den Volksklassen gehören. In den meisten Fällen liegt dies daran, dass Schüler aus den dominanten Klassen ein kulturelles Kapital haben, das andere nicht anhäufen konnten, nicht nur in der Schule, sondern auch in der kulturellen Bildung der Eltern, die ihren Kindern die Anforderungen nach Erhalt und Erweiterung ihrer privilegierten sozialen Situation beibringen würden. Kinder in Situationen sozialer Fragilität haben dieses Schutznetz nicht und haben in der Lage, viel größere Schwierigkeiten zu haben, weil sie nicht über das Wissen und die Praktiken verfügen, die von der vorherrschenden Kultur geschätzt werden. Sie würden sich nicht an den Werten beteiligen, die von diesen Eliten kultiviert werden, und würden aus diesem Grund dazu neigen, das Lernen der Schule als eine Form der Gewalt gegen ihre sozialen Werte und Praktiken zu betrachten, was die hohen Abbrecherquoten der brasilianischen Schule erklär[13]t.

In der Schule haben wir vielleicht die beste praktische Wahrnehmung von symbolischer Gewalt. Darin ist es zum Beispiel üblich, dass Schüler aus volkstümlichen Klassen auf eine Sprache, eine kulturelle Praxis und Werte stoßen, die sie nicht kennen, weil sie kein bestimmtes kulturelles Erbe haben, oder, wie Bourdieu betont, ein Kulturkapital, das es innen ermöglicht, sie zu verstehen oder zu assimilieren, ihre Schulleistung zu beeinträchtigen, zu einem Hindernis zu werden, das von der Institution selbst auferlegt wird, und die Leistung dieses Schülers zu 
delegitimieren. Trotzdem erkennt der Schüler nicht, dass seine geringe Schulleistung von sozialen Faktoren bestimmt wird und daher oft in misserfolgen endet, was sein Selbstwertgefühl in einem entscheidenden Moment in seiner nicht nur akademischen, sondern auch in menschlichen Ausbildungen beeinträchtigt.

Da wir als Bezugspunkt den Zugang und die Kontinuität im formalen Bildungssystem als eine der Hauptchancen einer Gesellschaft haben, die sich selbst als "meritokratisch" betrachtet, finden wir ein subtiles Beispiel dafür, wie die von der Sklavenvergangenheit auferlegten Beschränkungen verewigt, aber fast unsichtbar sind. Wenn wir in der Vergangenheit alles explizit ungleich wahrnehmen konnten, aufgrund des Phänotyps und des expliziten Ausschlusses von Sklaven zu Institutionen, scheint heute alles aufgrund der Schaffung eines öffentlichen Bildungssystems korrigiert zu sein, das alle Verantwortlichkeiten des Erfolgs oder des Versagens auf den individuellen Umfang verlagert. Es bedeckt die Sonne mit dem Sieb, [14]weil die meisten bedürftigen Bevölkerungen vom kulturellen Kapital ausgeschlossen sind, das bewusst von der Mittelschicht und den Eliten angeeignet wird, abgesehen vom Zugang zu einem ineffizienten öffentlichen Dienstleistungssystem.

In der kapitalistischen Welt verdammt inn der Mangel an materiellem Erfolg zum Status des Scheiterns. Stellen Sie sich vor, Sie sind dann ohne materielles Kapital und auch ohne Kulturkapital? Da die Formen des Klassenaufstiegs im räuberischen System des Kapitals nicht einfach sind, verewigt sich der Fortpflanzungszyklus von Armut und Ausgrenzung von Geburt an zu denen, die aus den ärmsten Klassen kommen. Dies wird dazu führen, dass das Thema, zusätzlich zu den geringeren Chancen, eine Formation zu bilden, die es inm ermöglichen wird, mit den Kindern der wohlhabenden Klassen zu konkurrieren, viel eher alle Arten von physischer und symbolischer Gewalt erleiden wird.

Da die Reproduktion der Klassenungleichheit aus der Wiege sowohl bewusst als auch unbewusst unterdrückt wird, ist es das Stereotyp des Schwarzen, leicht zu erkennen, das den zu schlachtenden und auszubeutenden Feind leicht identifiziert. Die "schwarze Gefahr", die 
jahrhundertelang als Passwort für das Massaker von Wehrlosen und Quilombola benutzt wurde, wird mit anderen Mitteln im offenen Massaker fortgesetzt und heute ohne Pejo von Armen und Schwarzen in Slums und Gefängnissen beklatscht. Und nicht nur das. Da es keine zeitliche Kontinuität zwischen der Sklaverei - die die Seele von innen zerstört, demütigt und erniedrigt, ihn zu einem Komplizen seiner eigenen Herrschaft macht - und der Produktion eines Geplänkels von Fehlanpassungen an die moderne Welt gab, erbten unsere Ausgeschlossenen, ohne eine Lösung der Kontinuität, allen Hass und feige Verachtung für die Schwächsten und mit weniger Fähigkeit, sich zu verteidigen. (Souza, 2019: 88).

Aber warum scheint eine solche Ungleichheit nicht von der brasilianischen Mittelschicht wahrgenommen zu werden? Wie wir am Anfang dieses Artikels sagten, reicht die Theorie der sozialen Klassen, die nach Einkommenskriterien genommen werden, nicht aus, um eine komplexe soziale Zusammensetzung wie die brasilianische zu verstehen, die durch die Folgen ihrer Sklavenvergangenheit geschwächt ist. Unserer Meinung nach ist es notwendig, die bloße wirtschaftliche Voreingenommenheit zu überwinden, um aus der Geschichte der Mentalitäten einzutrete[15]n. Ein Bürger der Mittelschicht neigt dazu, es als seine Klasse zu betrachten, so dass er keine instrumentale Grundlage für die Wahrnehmung des anderen hätte, was die Ausübung des Andersseins elend oder oft unerreichbar macht. Diese Abwesenheit von Dialektik produziert voreingenommene Menschen, ohne Wahrnehmung des anderen, in einem Land, das im 21. Jahrhundert in eine wettbewerbsfähige Marktordnung eingefügt wurde, die über den wirtschaftlichen Bereich hinausgeht und das Soziale, denken und wahrnehmende Welt erreicht. Der mentale Kolonialismus dominiert am Ende die Gemüter und behält gleichzeitig den Status quo bei.

\section{ABSCHLIEßENDE ÜBERLEGUNGEN}

Eine historische Rekonstitution der Bildung der brasilianischen Mittelschicht und ihres Archetyps von Vorurteilen zu verfolgen, ist aufgrund der Mehrdeutigkeit des Konzepts 
der sozialen Klasse selbst keine leichte Aufgabe. Der Faktor, den wir für dieses Verständnis für wesentlich halten, ist die vergangene Sklaverei, die dramatisch in das Verständnis der Gegenwart und des brasilianischen Sozialgefüges eingreift. Nur in der Langzeitserie können wir die hier vorgestellte Erzählung und unsere Argumentation für das Verständnis von Klassen aus dem soziokulturellen Kontext konstruieren und mit dem traditionellen Ökonomischen der Klassentheorien brechen, manchmal von liberalen Denker interpretiert, manchmal von marxistischen Denkern.

Wenn wir die Bedeutung der Sklavenvergangenheit für die Konfiguration von Klassenmentalitäten erkennen, die von sozialen Institutionen verewigt werden, können wir die Ungleichheiten und sozial konstituierten Stereotype naschieren, die ad infinitum zu reproduzieren scheinen.

Die Familie, die Schule, die Organisationen, all dies fördert im Laufe unserer Geschichte die Reproduktion der Privilegien elitärer Gruppen und der Mittelschicht, die mit der Tinte pseudowissenschaftlicher Analysen der Meritokratie getarnt ist, in einem der ungleichsten Länder der Welt. Der Trugschluss, dass es möglich ist, dass der fast angeborene Aufstieg für die einen zum Nachteil der anderen möglich ist, wurde so konsequent in das Imaginäre der Mittelschicht einfließen lassen, dass es inm gelang, sogar die populären Schichten zu durchdringen, in das Phänomen, mit dem wir uns im Text über die Introjection von Vorurteilen beschäftigen.

In der Mittelschicht und der oberen Mittelschicht geboren zu werden, ist fast gleichbedeutend mit der Reproduktion des Erfolgs vergangener Generationen und der Einbeziehung von Privilegien, die in zukunftdessen einen totalen Unterschied machen werden. Sie scheinen triviale Tatsachen zu sein, aber von klein auf die Disziplin des Studiums zu erwerben, bestimmte Umgebungen zu besuchen, mit Menschen mit dem kulturellen Kapital zu leben, die notwendig sind, um sich in sozialen Beziehungen zu profilieren, eine Fremdsprache im Schulwechsel zu lernen und gut ernährt zu werden, sind kleine Beispiele für Privilegien, die, zusätzlich zu Hunderten von anderen, den zukünftigen erfolgreichen Bürger prägen werden, als ob sein persönliches Verdienst das große Gefälle einer solchen Leistung gewesen wäre. 
Die Einbürgerung täglicher Gewalt gegen arme, schwarze Frauen und andere Minderheiten ist symptomatisch für die Gewalt, die die brasilianische Geschichte geprägt hat. Die Echos der Sklaverei sind in jedem Tod in der Favela zu hören, in jeder Aggression, die von der Polizei verübt wird, in jedem Witz am Sonntagmittag, der an den Tischen von Familien erzählt wird, die Rassismus bereits als etwas moralisch Akzeptables eingebürgert haben, als ob es die Menschenwürde nicht erniedrigen würde, wodurch die Realität der Mittelschicht in einen Käfig der Ängste, der Angst und des systemischen Hasses verwandelt wird.

\section{REFERENZEN}

BONFIM, Paulo Ricardo. Educar, higienizar e regenerar: uma história da eugenia no Brasil. São Paulo: Paco, 2019.

BOURDIEU, Pierre. A economia das trocas simbólicas. São Paulo: Perspectiva, 2004.

BRAUDEL, Fernand. Escritos sobre a história. São Paulo: Perspectiva, 1978.

CARVALHO, José Murilo de. Cidadania no Brasil: o longo caminho. São Paulo: Civilização Brasileira, 2003.

DOWBOR, Ladislau. A era do capital improdutivo. São Paulo: Editora Novas Palavras, 2017.

FAUSTO, Bóris. História do Brasil. 6. ed. São Paulo: Edusp/FDE, 1997. . História concisa do Brasil. São Paulo :Edusp, 2002.

FERNANDES, Florestan. Revolução burguesa no Brasil: ensaio de interpretação sociológica. Rio de Janeiro: Zahar, 1974.

FROMM, Erich. Anatomia da destrutividade humana. Rio de Janeiro: Zahar, 1975.

KENNETH, Maxwell. A devassa da devassa. São Paulo: Paz e Terra, 1995. 
KLEIN, Naomi. Sem logo: a tirania das marcas em um planeta vendido. Rio de Janeiro: Record, 2002.

PARSONS, Talcott. Social structure \& person. USA: Free Press, 2007.

PIAGET, Jean. A representação do mundo na criança. Curitiba: Ideias e Letras, 2005.

ROCHA, Sônia. Pobreza no Brasil. Rio de Janeiro: FGV, 2006.

SARTRE, Jean Paul. A prostituta respeitosa. Campinas: Papirus, 1992.

SOUZA, Jessé. A classe média no espelho. Rio de Janeiro: Estação Brasil, 2018. A elite do atraso. Rio de Janeiro: Estação Brasil, 2019.

SUCUPIRA, Eduardo. Introdução ao pensamento dialético. São Paulo: Alfa-Omega, 1984.

VOVELLE, Michel. Ideologias e mentalidades. São Paulo: Brasiliense, 1987.

WEBER, Max. A ética protestante e o espírito do capitalismo. São Paulo: Companhia das Letras, 2007.

YOUNG, Michael. The rise of meritocracy. United Kingdom: Transaction Pub, 1994 


\section{ANHANG A}

\begin{tabular}{|c|c|c|c|c|c|c|c|c|c|c|}
\hline \multirow{3}{*}{ Grandes Regiöes e car acteristicas selecionadas } & \multicolumn{10}{|c|}{$\begin{array}{l}\text { Taxa de frequência liquida a estabelecimento de ensino da população residente de } 6 \text { a } 24 \text { anos de idade, por grupos de idade e nivel de } \\
\text { ensino (\%) }\end{array}$} \\
\hline & \multicolumn{2}{|c|}{$\begin{array}{l}6 \text { a } 14 \text { anos, no ensino } \\
\text { fundamental }\end{array}$} & \multicolumn{2}{|c|}{$\begin{array}{l}6 \text { a } 10 \text { anos, nos anos } \\
\text { iniciais do ensino } \\
\text { fundamental }\end{array}$} & \multicolumn{2}{|c|}{$\begin{array}{l}11 \text { a } 14 \text { anos, nos anos } \\
\text { finais do ensino } \\
\text { fundamental }\end{array}$} & \multicolumn{2}{|c|}{$\begin{array}{l}15 \text { a } 17 \text { anos, no ensino } \\
\text { médio }\end{array}$} & \multicolumn{2}{|c|}{$\begin{array}{c}18 \text { a } 24 \text { anos, no ensino } \\
\text { superior }\end{array}$} \\
\hline & Taxa & CV (\%) & Taxa & $\mathrm{CV}(\%)$ & Taxa & CV (\%) & Taxa & CV (\%) & Taxa & CV (\%) \\
\hline Brasil & 97,0 & 0,1 & 95,5 & 0,2 & 85,9 & 0,3 & 68,5 & 0,6 & 23,2 & 1,3 \\
\hline Norte & 96,6 & 0,2 & 95,0 & 0,4 & 79,4 & 1,0 & 59,7 & 1,8 & 17,7 & 3,3 \\
\hline Nordeste & 96,8 & 0,1 & 95,3 & 0,2 & 82,0 & 0,6 & 60,7 & 1,1 & 16,8 & 2,9 \\
\hline Sudeste & 97,3 & 0,2 & 95,7 & 0,3 & 89,2 & 0,5 & 76,5 & 1,0 & 25,8 & 2,4 \\
\hline Sul & 97,4 & 0,2 & 96,1 & 0,3 & 89,6 & 0,6 & 69,6 & 1,4 & 29,1 & 2,4 \\
\hline Centro-Oeste & 96,8 & 0,3 & 95,0 & 0,4 & 88,4 & 0,9 & 70,4 & 1,7 & 30,1 & 2,9 \\
\hline Situaçäo do dom icilio & & & & & & & & & & \\
\hline Urbana & 97,1 & 0,1 & 95,5 & 0,2 & 87,2 & 0,3 & 70,6 & 0,7 & 25,6 & 1,4 \\
\hline Rural & 96,9 & 0,2 & 95,6 & 0,2 & 79,6 & 0,7 & 57,4 & 1,3 & 8,3 & 3,5 \\
\hline Sexo & & & & & & & & & & \\
\hline Homem & 96,9 & 0,1 & 95,3 & 0,2 & 83,5 & 0,5 & 63,6 & 0,9 & 19,7 & 1,8 \\
\hline Mulher & 97,2 & 0,1 & 95,7 & 0,2 & 88,3 & 0,4 & 73,7 & 0,7 & 26,8 & 1,5 \\
\hline Cor ou raça (1) & & & & & & & & & & \\
\hline Branca & 97,3 & 0,1 & 95,8 & 0,2 & 89,5 & 0,4 & 76,6 & 0,8 & 32,9 & 1,6 \\
\hline Preta ou parda & 96,9 & 0,1 & 95,3 & 0,2 & 83,7 & 0,4 & 63,5 & 0,9 & 16,7 & 1,8 \\
\hline $\begin{array}{l}\text { Classes de percentual de pessoas em ordem } \\
\text { crescente de rendimento domiciliar per capita }\end{array}$ & & & & & & & & & & \\
\hline Até $20 \%$ & 96,6 & 0,2 & 94,9 & 0,2 & 78,9 & 0,6 & 54,7 & 1,3 & 6,6 & 4,5 \\
\hline Mais de $20 \%$ até $40 \%$ & 96,9 & 0,2 & 95,4 & 0,3 & 85,3 & 0,6 & 65,3 & 1,2 & 12,0 & 3,3 \\
\hline Mais de $40 \%$ até $60 \%$ & 97,5 & 0,2 & 96,1 & 0,3 & 90,1 & 0,6 & 73,9 & 1,0 & 20,1 & 2,6 \\
\hline Mais de $60 \%$ até $80 \%$ & 97,5 & 0,3 & 95,9 & 0,5 & 93,0 & 0,6 & 81,1 & 1,2 & 32,3 & 2,1 \\
\hline Mais de $80 \%$ & 97,8 & 0,3 & 96,1 & 0,5 & 95,0 & 0,5 & 90,7 & 0,8 & 58,3 & 1,5 \\
\hline
\end{tabular}

Fonte: IBGE Pesquisa Nacional por Amostra de Domicilios Continua, 21 (1)

Nota. (1) Não são apresentados resultados para amarelos, indigenas e pessoas sem declaração de cor ou raça

\section{ANHANG B}

Distribuição percentual da população, por classes de percentual de pessoas em ordem crescente de rendimento real efetivo domicilia
cor ou raça, com indicação do coeficiente de variação, segundo as Grandes Regiöes e as Unidades da Federaça - 2017

\begin{tabular}{|c|c|c|c|c|c|c|c|c|c|c|c|c|}
\hline \multirow{4}{*}{$\begin{array}{l}\text { Grandes Regiöes e } \\
\text { Unidades da Federação }\end{array}$} & \multicolumn{12}{|c|}{ Distribuição percentual da populaçẫo, por classes de percentual de pessoas em ordem crescente de rendimento real efetivo domiciliar per capita e cor ou raça (\%) } \\
\hline & \multicolumn{4}{|c|}{ Total } & \multicolumn{4}{|c|}{ Entre os $10 \%$ com menores rendimentos } & \multicolumn{4}{|c|}{ Entre os $10 \%$ com maiores rendimentos } \\
\hline & \multicolumn{2}{|c|}{ Branca } & \multicolumn{2}{|c|}{ Preta ou parda } & \multicolumn{2}{|c|}{ Branca } & \multicolumn{2}{|c|}{ Preta ou parda } & \multicolumn{2}{|c|}{ Branca } & \multicolumn{2}{|c|}{ Preta ou parda } \\
\hline & Percentual & CV $(\%)$ & Percentual & CV (\%) & Percentual & CV (\%) & Percentual & CV (\%) & Percentual & CV $(\%)$ & Percentual & $\mathrm{CV}(\%)$ \\
\hline Brasil & 43,6 & 0,5 & 55,4 & 0,4 & 23,9 & 1,8 & 75,2 & 0,6 & 71,7 & 0,8 & 26,3 & 2,3 \\
\hline Norte & 20,1 & 1,9 & 78,4 & 0,5 & 12,5 & 6,8 & 84,8 & 1,3 & 34,2 & 3,8 & 64,4 & 1,9 \\
\hline Rondônia & 31,2 & 3,0 & 68,2 & 1,4 & 23,6 & 9,3 & 75,2 & 2,9 & 40,9 & 7,9 & 58,4 & 5,5 \\
\hline Acre & 19,1 & 4,3 & 79,9 & 1,1 & 14,1 & 12,5 & 85,3 & 2,1 & 31,8 & 8,2 & 67,7 & $3,8>>$ \\
\hline Amazonas & 18,1 & 4,7 & 79,5 & 1,2 & 11,0 & 13,1 & 86,2 & 2,0 & 38,2 & 9,8 & 60,5 & 5,8 \\
\hline Roraima & 21,5 & 5,6 & 72,4 & 2,0 & 14,6 & 17,7 & 71,3 & 7,3 & 40,9 & 8,4 & 55,5 & $6,5>>1$ \\
\hline Pará & 18,2 & 3,3 & 80,6 & 0,8 & 11,8 & 12,6 & 85,8 & 2,2 & 30,0 & 5,5 & 68,9 & 2,4 \\
\hline Amapá & 19,3 & 6,5 & 80,3 & 1,6 & 10,6 & 26,6 & 87,7 & 3,6 & 25,9 & 13,4 & 73,4 & $4,8>$ \\
\hline Tocantins & 23,7 & 4,7 & 75,3 & 1,5 & 14,8 & 19,0 & 84,4 & 3,4 & 42,5 & 6,4 & 56,2 & 4,7 \\
\hline Nordeste & 24,8 & 1,2 & 74,6 & 0,4 & 18,7 & 3,3 & 80,8 & $\mathbf{0 , 8}$ & 42,8 & 2,7 & 56,4 & 2,0 \\
\hline Maranhäo & 18,5 & 2,6 & 80,8 & 0,6 & 15,7 & 6,9 & 83,9 & 1,3 & 31,5 & 5,5 & 67,4 & 2,6 \\
\hline Prauí & 20,5 & 4,8 & 79,4 & 1,2 & 18,6 & 11,0 & 81,2 & 2,5 & 40,9 & 7,6 & 58,6 & 5,5 \\
\hline Ceará & 27,5 & 2,2 & 71,9 & 0,8 & 20,4 & 6,2 & 79,1 & 1,6 & 46,9 & 4,3 & 52,6 & 3,8 \\
\hline Rio Grande do Norte & 36,7 & 2,6 & 63,1 & 1,5 & 30,6 & 7,1 & 69,1 & 3,1 & 50,9 & 5,5 & 48,8 & 5,8 \\
\hline Paraba & 33,8 & 3,0 & 66,1 & 1,5 & 30,1 & 7,2 & 69,8 & 3,1 & 54,1 & 5,4 & 45,7 & 6,3 \\
\hline Pernambuco & 30,7 & 3,0 & 68,5 & 1,4 & 23,2 & 8,3 & 76,5 & 2,5 & 51,5 & 5,8 & 47,4 & 6,2 \\
\hline Alagoas & 23,4 & 2,8 & 76,1 & 0,9 & 21,8 & 6,3 & 77,9 & 1,8 & 36,1 & 6,6 & 63,5 & 3,8 \\
\hline Sergipe & 20,8 & 5,4 & 78,3 & 1,4 & 13,6 & 13,9 & 85,7 & 2,2 & 36,6 & 12,4 & 61,7 & 7,3 \\
\hline Bahia & 19,2 & 3,9 & 80,2 & 0,9 & 14,7 & 9,4 & 84,6 & 1,6 & 35,3 & 9,1 & 63,6 & 4,9 \\
\hline Sudeste & 51,2 & 0,9 & 47,6 & 1,0 & 35,2 & 2,8 & 64,2 & 1,5 & 78,1 & 1,1 & 19,0 & 4,6 \\
\hline Minas Gerais & 40,4 & 1,7 & 59,3 & 1,2 & 24,5 & 5,5 & 75,2 & 1,8 & 64,8 & 2,6 & 34,8 & 4,8 \\
\hline Espirito Santo & 39,2 & 2,6 & 60,2 & 1,7 & 22,5 & 10,6 & 77,2 & 3,1 & 64,5 & 3,0 & 35,2 & 5,6 \\
\hline Rio de Janeiro & 43,8 & 1,7 & 55,7 & 1,3 & 28,4 & 5,2 & 71,2 & 2,1 & 74,5 & 1,9 & 24,8 & $5,8>$ \\
\hline São Paulo & 60,1 & 1,2 & 38,1 & 2,0 & 46,8 & 3,7 & 52,4 & 3,3 & 83,1 & 1,4 & 12,3 & 9,0 \\
\hline Sul & 75,6 & 0,5 & 23,7 & 1,7 & 59,3 & 2,1 & 39,9 & 3,2 & 91,3 & 0,6 & 7,9 & 6,9 \\
\hline Paraná & 67,2 & 1,1 & 31,7 & 2,3 & 52,2 & 3,8 & 46,7 & 4,3 & 86,7 & 1,3 & 11,4 & 9,4 \\
\hline Santa Catarina & 82,8 & 0,7 & 16,9 & 3,4 & 68,5 & 2,9 & 31,2 & 6,4 & 92,8 & 0,9 & 6,8 & 11,7 \\
\hline Rio Grande do Sul & 79,6 & 0,9 & 20,0 & 3,5 & 64,2 & 3,3 & 35,2 & 6,1 & 94,4 & 0,9 & 5,5 & 15,4 \\
\hline Centro-Oeste & 36,3 & 1,4 & 62,6 & 0,8 & 25,0 & 4,5 & 74,0 & 1,5 & 56,2 & 3,4 & 41,0 & 4,0 \\
\hline Mato Grosso do Sul & 43,2 & 2,6 & 55,5 & 2,1 & 28,9 & 8,7 & 69,1 & 3,7 & 65,0 & 3,6 & 34,1 & 6,8 \\
\hline Mato Grosso & 31,8 & 3,2 & 67,0 & 1,5 & 23,7 & 12,3 & 74,9 & 3,9 & 54,4 & 4,8 & 43,9 & 6,0 \\
\hline Goiás & 35,7 & 2,2 & 63,6 & 1,2 & 23,9 & 7,4 & 75,8 & 2,3 & 51,6 & 4,2 & 47,7 & 4,6 \\
\hline Distrito Federal & 36,7 & 4,1 & 61,6 & 2,4 & 22,8 & 11,0 & 76,5 & 3,3 & 59,3 & 10,5 & 29,1 & 10,0 \\
\hline
\end{tabular}

Fonte: IBGE Pesquisa Nacional por Amostra de Domic lios Contínua, 2017 consolidado de primeiras entrevistas

Notas: Exclusive as pessoas cuja condição no arranjo domiciliar era pensionista, empregado domés tico ou parente do empregado doméstico.

Rendimentos deflacionados para reais médios do próprio ano.

Não são apresentados resultados para amarelos, indigenas e pessoas sem declaraçâo de cor ou raça

Disponível em: https://www.nucleodoconhecimento.com.br/geschichte/archetyp-us-vorurteile 


\section{ANHANG C - FOOTNOTE REFERENZEN}

2. Para o entendimento da financeirização do capitalismo e suas repercussões no mundo do trabalho, vide DOWBOR, Ladislau. A era do capital improdutivo. São Paulo: Editora Novas Palavras, 2017.

3. SARTRE, Jean Paul. A prostituta respeitosa. Campinas: Papirus, 1992. In diesem Stück schildert der Autor, wie die vom Opfer eingearbeitete Introjection von Vorurteilen in die Gesellschaft der südlichen Vereinigten Staaten eingebürgert wird.

4. BRAUDEL, Fernand. Escritos sobre a história. São Paulo: Perspectiva, 1978.

5. FROMM, Erich. Anatomia da destrutividade humana. Rio de Janeiro: Zahar, 1975.

6. Uma das obras mais consistentes e rica em fontes primárias sobre a organização das regiões mineradoras é de KENNETH, Maxwell. Die Ausschweifung der Ausschweifung. São Paulo: Paz e Terra, 1995.

7. Sobre o período em questão, vide FAUSTO, Bóris. História concisa do Brasil. São Paulo: Edusp, 2002.

8. Para um melhor entendimento sobre a construção do processo da cidadania brasileira, vide CARVALHO, José Murilo de. Cidadania no Brasil: o longo caminho. São Paulo: Civilização Brasileira, 2003.

9. Wir verwendeten metaphorisch den Ausdruck "bewölkter Schneeball", weil Schwarze, Mestizen, Indigene, Braune, neben natürlich der marginalisierten weißen Bevölkerung, das Kontingent der getrennten "Schlacke" aus der brasilianischen hierarchischen Gesellschaft verdickten. Während des 19. Jahrhunderts, Wissenschaftlicher Rassismus und die Prinzipien der Eugenik in Amerika gefunden, vor allem in amerikanischen Eugeniker, seine wichtigsten Sprecher. Obwohl Vorurteile in Brasilien fälschlicherweise auf phänotypische Themen beschränkt sind, ist die Rolle der Eugeniker bei der Stimulierung der Bleichenpolitik, die von der brasilianischen Regierung bewusst angenommen wurde, um die Genetik der Bevölkerung zu reinigen, 
beträchtlich. Dies trug direkt zum Anreiz für Rassismus und Tierisierung von Personen bei, die als "minderwertig" gelten. BONFIM, Paulo Ricardo. Educar, higienizar e regenerar: uma história da eugenia no Brasil. São Paulo: Paco, 2019.

10. In Brasilien durchdringt das Konzept der "Meritokratie" den gesunden Menschenverstand und die Demagogie. Aus unserer Sicht wird es nur dann als "Meritokratie" verstanden, wenn wir ihren Ursprung in der Literatur eingeben, in der das Konzept auf satirische und ironische Weise geboren wird. Ver YOUNG, Michael. The rise of meritocracy. United Kingdom: Transaction Pub, 1994.

11. "Mit dem Aufkommen der Massenkultur im 20. Jahrhundert schafft der Kapitalismus eine neue Symbolik falscher Inklusion, indem er das in die heutige Gesellschaft einführt, was wir die "Unterdrückung der Subjektivität" nennen, und die Entwicklung des so genannten "mimetischen Verhaltens" anhebt, das heißt, das Individuum hätte aufgehört, eine eigene Persönlichkeit zu entwickeln, die inn in Konflikt und Spannung mit der etablierten gesellschaftlichen Realität bringen würde." KLEIN, Naomi (2009). Sem logo: a tirania das marcas em um planeta vendido. Rio de Janeiro: Record, 2002.

12. Tomamos como referência o conceito de status em WEBER, Max. Protestantische Ethik und der Geist des Kapitalismus. São Paulo: Companhia das Letras, 2007.

13. Nach den Anhängen $A$ und $B$ können wir die historischen Missstände des Prozesses der Ausgrenzung schwarzer und brauner Bevölkerungsgruppen auf ihrem Bildungsweg deutlich feststellen, indem wir diese Gruppen von den Mechanismen des Zugangs abbringen, die die heutige Gesellschaft für ein würdevolles Leben benötigt, das eine bessere berufliche Vermittlung erfordert, ihre Einkommen und ihre Fähigkeit, in die kapitalistische Welt einzutreten, beeinträchtigt.

14. Brasilianische populäre Ausdruck verwendet, um ineffektive Maßnahmen für bestimmte Probleme zu beschreiben.

15. VOVELLE, Michel. Ideologias e mentalidades. São Paulo: Brasiliense, 1987. 
Gesendet: Dezember, 2019.

Genehmigt: Februar 2020. 